(1)

CrossMark

\title{
Defining asthma and assessing asthma outcomes using electronic health record data: a systematic scoping review
}

\author{
Mohammad A. Al Sallakh (1) ${ }^{1,2}$, Eleftheria Vasileiou ${ }^{2,3}$, Sarah E. Rodgers ${ }^{1,4}$, \\ Ronan A. Lyons ${ }^{1,4}$, Aziz Sheikh ${ }^{2,3,4}$ and Gwyneth A. Davies ${ }^{1,2}$
}

Affiliations: ${ }^{1}$ Swansea University Medical School, Swansea, UK. ${ }^{2}$ Asthma UK Centre for Applied Research, UK. ${ }^{3}$ Usher Institute of Population Health Sciences and Informatics, The University of Edinburgh, Edinburgh, UK. ${ }^{4}$ The Farr Institute of Health Informatics Research, UK.

Correspondence: Mohammad A. Al Sallakh, Data Science Building, Swansea University, Singleton Park, Swansea, SA2 8PP, UK. E-mail: M.A.AlSallakhdswansea.ac.uk

@ERSpublications

Inconsistent/underreported asthma case definitions are common in research, limiting reproducibility/ comparability http://ow.ly/knmc30a0ltp

Cite this article as: Al Sallakh MA, Vasileiou E, Rodgers SE, et al. Defining asthma and assessing asthma outcomes using electronic health record data: a systematic scoping review. Eur Respir J 2017; 49: 1700204 [https://doi.org/10.1183/13993003.00204-2017].

ABSTRACT There is currently no consensus on approaches to defining asthma or assessing asthma outcomes using electronic health record-derived data. We explored these approaches in the recent literature and examined the clarity of reporting.

We systematically searched for asthma-related articles published between January 1, 2014 and December 31, 2015, extracted the algorithms used to identify asthma patients and assess severity, control and exacerbations, and examined how the validity of these outcomes was justified.

From 113 eligible articles, we found significant heterogeneity in the algorithms used to define asthma ( $n=66$ different algorithms), severity $(n=18)$, control $(n=9)$ and exacerbations $(n=24)$. For the majority of algorithms $(n=106)$, validity was not justified. In the remaining cases, approaches ranged from using algorithms validated in the same databases to using nonvalidated algorithms that were based on clinical judgement or clinical guidelines. The implementation of these algorithms was suboptimally described overall.

Although electronic health record-derived data are now widely used to study asthma, the approaches being used are significantly varied and are often underdescribed, rendering it difficult to assess the validity of studies and compare their findings. Given the substantial growth in this body of literature, it is crucial that scientific consensus is reached on the underlying definitions and algorithms.

This article has supplementary material available from erj.ersjournals.com

Received: Jan 272017 | Accepted after revision: March 092017

Support statement: This work was funded by Health and Care Research Wales and Abertawe Bro Morgannwg University Health Board. It was carried out with the support of the Asthma UK Centre for Applied Research (AUK-AC-2012-01). We also acknowledge the support from The Farr Institute of Health Informatics Research. The Farr Institute is supported by a 10-funder consortium: Arthritis Research UK, the British Heart Foundation, Cancer Research UK, the Economic and Social Research Council, the Engineering and Physical Sciences Research Council, the Medical Research Council, the National Institute of Health Research, the National Institute for Social Care and Health Research (Welsh Assembly Government), the Chief Scientist Office (Scottish Government Health Directorates), and the Wellcome Trust (Medical Research Council grants CIPHER MR/K006525/1, Scotland MR/K007017/1). Funding information for this article has been deposited with the Crossref Funder Registry.

Conflict of interest: Disclosures can be found alongside this article at erj.ersjournals.com

Copyright OERS 2017 


\section{Introduction}

Asthma is in clinical practice a diagnosis based on patient history, examination and objective tests [1]. It is, however, increasingly considered to represent a heterogeneous group of disorders with different phenotypes and endotypes [2]. The clinical definitions of asthma and its key outcomes, including disease severity, control and attacks/exacerbations, have been the subject of vigorous debate [3-8].

Particular challenges arise in the context of epidemiological studies where validated operational definitions are needed $[9,10]$. These studies are, increasingly, being undertaken using electronic health record (EHR)-derived data, which adds a further layer of complexity as the use of valid and reliable approaches is essential to ensure the reproducibility of research findings [11].

In order to assess current approaches, we systematically interrogated the recent EHR-based asthma literature. Our specific objectives were to: 1) describe the different methods of defining asthma and assessing disease severity, control and exacerbations in EHR-based studies; 2) investigate whether authors reported on the validity of those methods; and 3) assess their reporting practices.

\section{Methods}

We conducted a systematic scoping review based on the ARKSEY and O'MALLEY [12] five-stage framework, including identifying the research question, identifying relevant studies, study selection, data charting, and collating, summarising and reporting the results. The research questions were: 1) how were asthma and its key outcomes defined using EHR data in the recent literature, 2) how did authors report on the validity of their EHR-based algorithms and 3) how clearly were the EHR-related methods reported?

\section{Eligibility criteria and search strategy}

We searched PubMed using a broad query (supplementary table E1) to retrieve asthma studies that used EHR-derived data and were published between January 1, 2014 and December 31, 2015. The search query was iteratively improved by adding many variations and equivalents of the keywords "EHR" and "routinely collected data" as well as named data sources found in the literature. Only articles written in English were included.

\section{Study selection}

We excluded nonrelevant articles by reviewing titles and abstracts, referring to the full text when needed. We included only articles where asthma was a main finding. For the purpose of this scoping review, we limited the concept of EHR-derived data to coded, objective, individual-level data that were generated as a byproduct of routine healthcare.

\section{Data extraction and synthesis}

From each of the eligible articles, we extracted and summarised information from the full text and online supplements, including basic bibliography, setting (country) and design; names and types of EHR-derived data sources used; algorithms to identify asthma patients, assess disease severity, assess control and define exacerbation; and how authors reported on algorithm validity. In this context, we referred to "validation" as any attempt to assess the algorithm's concurrent or construct validity. We used the RECORD Statement's 13-item checklist to assess the clarity of reporting of other EHR-related aspects such as clinical code lists used in the algorithms and the implications of using EHR data in asthma research. The RECORD Statement is a recently introduced extension to the STROBE Statement which helps improve the reporting of observational studies conducted using routinely collected data [13]. Supplementary table E2 describes the data extraction and charting tool. Article screening and data extraction were performed independently by two authors (M.A. Al S. and E.V.), with a third author arbitrating (G.A.D.).

\section{Role of the funding sources}

The funding sources had no role in study design, in the collection, analysis and interpretation of data, in the writing of the report, or in the decision to submit the paper for publication.

\section{Results}

We included 113 articles in the scoping review. Figure 1 shows the study selection process. Most studies were conducted in the USA, Taiwan and Canada (supplementary table E3), and employed longitudinal designs (supplementary table E4). The most commonly used data types were health insurance claims, followed by medical record repositories and dispensing databases (supplementary table E5). 
FIGURE 1 Flowchart for study selection in this scoping review.

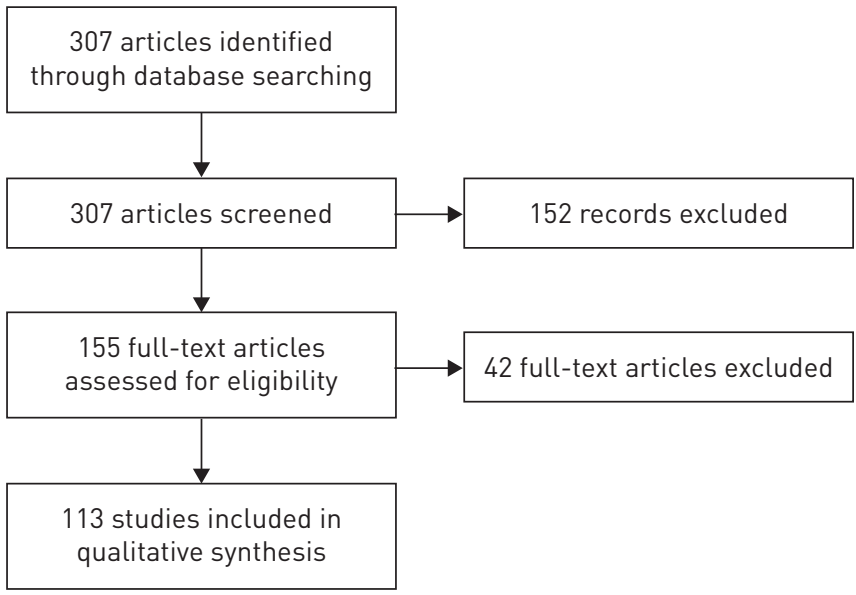

"Persistent asthma" was defined over 12 and 24 months using the US Healthcare Effectiveness Data and Information Set (HEDIS) criteria [14], which involved assessing for any of the following asthma-related events: 1) emergency department visit, 2) hospitalisation, 3) outpatient visit and two asthma prescriptions or 4) four asthma prescriptions [15-18]; by HEDIS criteria except "four asthma prescriptions" [19]; and by any asthma encounter (hospitalisation or emergency department visit) or using oral corticosteroids (OCSs) for $\geqslant 3$ days [20].

"Current asthma" was defined by any asthma encounter in the last 3 years [21].

"Current general practitioner-reported and diagnosed asthma" was defined as any asthma encounter in the last 12 months; "current general practitioner-reported, diagnosed and treated asthma" as the same plus any asthma prescription in the same period [22].

Patients with treated asthma were otherwise required to have at least three dispensing events of asthma treatments in three different quarters of the year [23].

"Acute asthma" was defined using any asthma diagnosis codes in emergency department or inpatient data [24].

In the remaining studies, the label "asthma" was defined using various algorithms, some of which were similar to those of the aforementioned more specific labels.

The intervals over which asthma diagnostic/management and prescription codes where queried were specified in 31 and eight studies, respectively. The positions of diagnostic codes in the encounter (i.e. primary or secondary) were specified in 37 studies.

We identified the following approaches in these algorithms: requiring diagnostic/management events, prescription events or both (supplementary table E7). In addition, to exclude likely nonasthma patients, some studies applied additional nonasthma criteria to restrict the study population based on age (supplementary table E8) and/or comorbidities (supplementary table E9).

\section{Assessing asthma severity}

18 studies used 20 different algorithms to assess asthma severity (supplementary table E10) as binary (i.e. severe versus nonsevere asthma) $[15,23,25-38]$ or ordinal variables (mild, moderate and severe asthma [39]; low-, moderate- and high-risk asthma [40]). The algorithms were based on one or more of the following asthma-related variables: number and/or dosage of prescriptions (i.e. short-acting $\beta_{2}$-agonist (SABA), inhaled corticosteroid, OCS and leukotriene receptor antagonist), and number of hospitalisations, emergency department and outpatient visits. Almost all algorithms $(n=17)$ used prescriptions (either alone or with other variables), while one algorithm was based only on hospitalisations and emergency department visits [36]. The intervals over which asthma severity was assessed were 3 [29], 6 [38], 12 $[15,23,28,30-32,34,36,37,39,40]$ or 24 months [33, 35], or unclear [26, 27].

\section{Assessing asthma control}

Nine studies assessed asthma control using 11 algorithms, in nine of which the interval was 12 months, in one 1-3 months and in the remaining study this was unclear (supplementary table E12). Uncontrolled asthma was defined by a minimum number/dose of SABA prescriptions [30, 31, 39, 41, 42]; any or short-course OCS prescriptions [30, 31, 41-44]; any hospitalisation or emergency department visit with either diagnosis of asthma [27, 30, 31, 41-43, 45] or (in already diagnosed asthma patients) diagnosis of 
status asthmaticus, pneumonia, dyspnoea or respiratory insufficiency [30]; unscheduled outpatient visits for asthma or lower respiratory tract infections (LRTIs) [31]; and general practitioner consultations for LRTIs requiring antibiotics in asthma patients [31]. Asthma impairment was defined based on the required SABA use, namely an average of more than two salbutamol puffs per day [31]. One study assessed asthma control based on number of OCS and SABA prescriptions per year (without giving any further details about the actual algorithm) [41].

\section{Defining exacerbations}

24 studies defined exacerbations using EHR-derived data (supplementary table E11) as a dichotomous variable (absent versus present) [16, 17, 23, 27, 30-32, 35, 37-39, 42-44, 46-54] or stratified into absent, moderate and severe [55]. OCS prescriptions were used as a marker for exacerbations in 17 studies, either alone $[23,30,31,35,39,42,47,48,53]$ or with a concurrent asthma encounter (e.g. a general practice, outpatient or emergency department visit, or hospitalisation within 5 or 7 days) $[16,17,32,37,38,46,52$, 54]. In one study, exacerbations were defined by a minimum of six SABA prescriptions per year [47]. Other definitions included an outpatient code of "asthma exacerbation" [52], asthma hospitalisation [23, $30,32,35,37,39,43,44,46,48,50,51,53-55]$, asthma emergency department visit $[16,30-32,35,37,38$, $43,44,46,48,51-54]$, or hospitalisation with diagnosis of status asthmaticus or (in already diagnosed asthma patients) diagnosis of pneumonia, dyspnoea or respiratory insufficiency [30].

\section{Clarity of reporting}

Overall, the reporting of methodological aspects of using EHR-derived data was suboptimal. The majority of studies presented no information on algorithm validity. Among studies that reported on the validity, we identified 10 practices of reporting or justifying on the validity of algorithms (table 1): 1) performing validation or concordance analysis in the same study against other measures based on different data sources (e.g. medical record review or patient-reported measures); 2) referring to previous validation of similar algorithms in the same or 3) different databases; 4) referring to previous validation of similar algorithms for different diseases in the same or 5) different database; 6) using algorithms "consistent" with previous studies in the same or 7) different databases; 8) using nationally developed algorithms; 9) using algorithms based on clinical guidelines; and 10) relying on previous validation of the database content. Some studies did not provide clear algorithms for asthma severity or control, but only referred to their components [23, 35, 37, 38, 41].

Of the 113 reviewed studies, 40 studies used record linkage, of which 17 mentioned it in the abstract and 28 provided at least some explanation in the full text. The geographical region, time frame of data and types or names of the data sources were mentioned in 83, 91 and 104 abstracts, respectively. 83 studies reported their extent of access to the data sources. The intervals over which the algorithms were applied were often not reported. 111 studies touched on the implications of using EHR data to study asthma. Of these, 64 and 63 studies discussed the risk of misclassification bias and unmeasured confounding, respectively. Six studies acknowledged the possible changes over time in data quality and coding practices, and the entailing changes in case definition eligibility and accuracy. Five studies explained their data cleansing procedures. Finally, no study shared the programming codes of data preparation and analysis.

TABLE 1 Practices of reporting or justifying the validity of algorithms to define and assess asthma using electronic health record-derived data

Algorithm validity was justified by

\begin{tabular}{ccccc}
\multicolumn{5}{c}{ Algorithms $\mathrm{n}$} \\
\hline Identifying & $\begin{array}{c}\text { Assessing } \\
\text { severity }\end{array}$ & $\begin{array}{c}\text { Assessing } \\
\text { control }\end{array}$ & $\begin{array}{c}\text { Defining } \\
\text { exacerbation }\end{array}$ & $\begin{array}{c}\text { Total per } \\
\text { category }\end{array}$
\end{tabular}

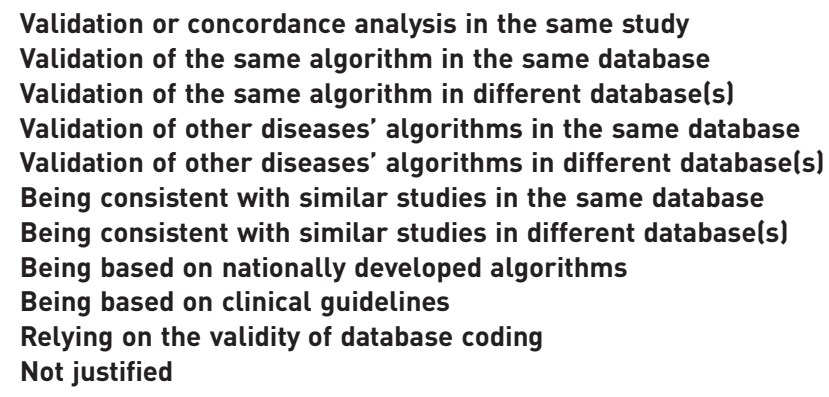

4

$\begin{array}{cc}4 & 0 \\ 14 & 1 \\ 2 & 6 \\ 2 & 0 \\ 1 & 0 \\ 1 & 0 \\ 1 & 0 \\ 3 & 0 \\ 0 & 3 \\ 5 & 0 \\ 76 & 8\end{array}$

$\left.\begin{array}{ll}0 \\ 1 \\ 6 \\ 0\end{array}\right)$

$\begin{array}{lcc}0 & 0 & 4 \\ 1 & 1 & 17 \\ 3 & 2 & 13 \\ 0 & 0 & 2 \\ 0 & 0 & 1 \\ 1 & 0 & 2 \\ 0 & 1 & 2 \\ 0 & 2 & 5 \\ 0 & 0 & 3 \\ 0 & 0 & 5 \\ 4 & 18 & 106\end{array}$




\section{Discussion}

Statement of principal findings

This systematic analysis of the contemporaneous asthma literature found evidence of considerable international activity in using EHR-derived data to study a variety of asthma populations and outcomes. Importantly, we also found wide variations in the approaches used, with limited attention being paid to the validity of the underlying algorithms used and suboptimal reporting of studies. This poses a major challenge to the interpretation and reproducibility of this important, emerging body of research inquiry.

\section{Strengths and limitations}

To the best of our knowledge, this is the first systematic exercise to investigate the quality of reporting on EHR-based studies, especially the validity of measures, in the context of asthma. In undertaking this work, we used robust approaches which involved two people independently selecting studies and undertaking data extraction. The findings may also apply to other chronic diseases. This scoping review had no geographic limits, but it was confined to assessing the recent literature. Examining the most recent asthma literature is most likely to provide meaningful insights on current practices. A limitation is that we did not systematically check whether the references provided to support the claimed validity of algorithms in question actually provided sufficient evidence of validity. For example, differences might exist between the algorithms used in a given study and those previously validated.

\section{Interpretation in the light of previous studies}

Although EHR-derived data are convenient resources for research, they are originally collected for other purposes, and usually suffer from missing or incorrect data and potential biases [56-58]. In addition, EHR systems usually fail to capture complete and accurate clinical information at the point of care due to design limitations and inefficient use of these systems by clinicians to document clinical data [59, 60].

These issues impose challenges on their use to assess a complex and heterogeneous condition such as asthma. For example, asthma diagnosis codes, which are commonly used solely for patient identification, may be recorded after a trial or wrong diagnosis and do not capture undiagnosed patients [61]. In addition, many EHR-derived databases often lack important variables, such as lung function, indication of dispensed medications, adherence to treatment and lifestyle, which are vital for identifying and assessing asthma patients. These challenges are, however not insurmountable. In this scoping review, we found several techniques intended to improve algorithm accuracy, such as age limitation, comorbidity exclusion and diagnosis position restriction.

Ideally, algorithms should be validated in the databases in which they are used. However, this was often not the case. Instead, using algorithms with only reasonable face validity based on clinical guidelines or clinical judgement is a very common practice in EHR-based studies [62, 63]. These approaches assume that clinical codes in the database accurately represent the patient's actual healthcare events [62].

Underreporting on implementation details and methods' validity compromises transparency and reproducibility, a crucial issue in medical research. It has been previously found that in EHR-based studies, full lists of clinical codes were often not reported [64]. A recent, large-scale reproducibility exercise identified similar challenges due to suboptimal reporting of EHR-based studies, particularly sharing code lists and algorithms [65].

The significant methodological heterogeneity we found in EHR-based asthma assessment algorithms reflects, in addition to the content differences between the databases used, the lack of consensus on the clinical definitions in the first place despite continuous standardisation efforts $[5,6,66,67]$. The focus of our work was to examine asthma definitions and their validity specifically in the context of EHR, but this highlights the fundamental need to reach consensus on clinical asthma definitions and the appropriate validation of asthma diagnosis. For example, there is still an active debate on whether lung function is essential to establish asthma diagnosis $[7,8]$. A recent study also found significant variation in algorithms to assess asthma severity from health insurance data [68]. Unjustified interstudy variation in the operational definitions of the same clinical concepts creates challenges for comparability, meta-analysis and evidence synthesis. These issues have been raised for asthma [69] and other allergic conditions, such as peanut allergy [70, 71] and anaphylaxis [72], where wide variations in findings were potentially attributed to inconsistent case definitions.

Implications for policy, practice and research

This scoping review sheds light on the opportunities offered by the increasingly ubiquitous EHRs, but also highlights considerable heterogeneity and suboptimal reporting of EHR-based asthma assessment algorithms and the implications of these practices on comparability and reproducibility of studies. 
Developing reliable algorithms to assess asthma outcomes using EHR data is a nontrivial challenge. In addition, standardising such algorithms across different populations may be impractical since databases differ in content, validity may not hold across different populations and no best practice currently exists [68]. Similar challenges arise when comparing asthma epidemiology between multiple populations [73]. These methodological issues, in addition to suboptimal reporting, should be considered when interpreting and synthesising evidence from geographically dispersed studies.

With the accelerating availability of EHR-derived data and their use to study asthma, we believe that consideration needs to be given to convening an international task force to work on the harmonisation of those algorithms under uniform and consistent clinical labels, while considering the differences between populations and databases. In addition, validation of these algorithms in the respective populations should be given a high priority. Furthermore, to allow more accurate assessment of asthma from EHR data, efforts are needed to improve the capture and coding of asthma-related data at the point of care [74], which requires more efficient EHR systems $[59,60]$. In addition, emerging data sources such as patient-generated data and wearables need to be harnessed [75]. Finally, to improve the clarity of reporting on EHR-related methodological aspects, we strongly advocate the adoption of the RECORD Statement as an extension of the STROBE Statement by both authors and journal editors [13]. Optimal reporting should include complete code lists, detailed algorithms and validity assessment. Implications of using EHR-derived data to study a complex condition such as asthma should be clearly communicated to enable judgement of internal and external validity.

In summary, we found that there is considerable international interest in exploiting EHR-derived data to study asthma, but that there are considerable variations in the approaches used. These variations are compounded by suboptimal reporting of methods, which makes it difficult to assess the reproducibility of research. Given the substantial investments taking place in EHRs globally, this body of work is likely to grow significantly in the coming years. It is therefore important that the asthma-interested research community works to place it on a solid footing in order to ensure the quality and reproducibility of this work.

\section{Acknowledgements}

Author contributions: M.A. Al Sallakh, S.E. Rodgers, G.A. Davies, A. Sheikh and R.A. Lyons developed the concept and methods. M.A. Al Sallakh conducted the literature search. M.A. Al Sallakh and E. Vasileiou independently reviewed the studies with G.A. Davies arbitrating. All authors contributed to the development of methods, interpretation of findings and manuscript writing, and critically reviewed and approved the final manuscript.

\section{References}

1 Global Initiative for Asthma. Global Strategy for Asthma Management and Prevention. 2015. www.ginasthma.org, Date last accessed: October 212015.

Wenzel SE. Asthma phenotypes: the evolution from clinical to molecular approaches. Nat Med 2012; 18: 716-725. Hargreave FE, Nair P. The definition and diagnosis of asthma. Clin Exp Allergy 2009; 39: 1652-1658.

A plea to abandon asthma as a disease concept. Lancet 2006; 368: 705.

Bousquet J, Mantzouranis E, Cruz AA, et al. Uniform definition of asthma severity, control, and exacerbations: document presented for the World Health Organization Consultation on Severe Asthma. J Allergy Clin Immunol 2010; 126: 926-938.

6 Reddel HK, Bateman ED, Becker A, et al. A summary of the new GINA strategy: a roadmap to asthma control. Eur Respir J 2015; 46: 622-639.

7 Akker IL, van der Zeijden H, Verheij TJ. Is spirometry essential in diagnosing asthma? Yes. Br J Gen Pract 2016; 66: 484-484.

8 Levy ML. Is spirometry essential in diagnosing asthma? No. Br J Gen Pract 2016; 66: 485-485.

9 Toelle BG, Peat JK, Salome CM, et al. Toward a definition of asthma for epidemiology. Am Rev Respir Dis 1992; 146: 633-637.

10 Pekkanen J, Pearce N. Defining asthma in epidemiological studies. Eur Respir J 1999; 14: 951-957.

11 Ioannidis JP. Why most published research findings are false. PLoS Med 2005; 2: e124.

12 Arksey H, O’Malley L. Scoping studies: towards a methodological framework. Int J Soc Res Methodol 2005; 8: 19-32.

13 Benchimol EI, Smeeth L, Guttmann A, et al. The REporting of studies Conducted using Observational Routinely-collected health Data (RECORD) Statement. PLoS Med 2015; 12: e1001885.

14 National Committee for Quality Assurance. Use of Appropriate Medications for People with Asthma: HEDIS 2003. Vol. 2. Washington, National Committee for Quality Assurance, 2003; pp. 25-28.

15 Wu CL, Andrews AL, Teufel RJ, et al. Demographic predictors of leukotriene antagonist monotherapy among children with persistent asthma. J Pediatr 2014; 164: 827-831.

16 Zeiger RS, Schatz M, Li Q, et al. High blood eosinophil count is a risk factor for future asthma exacerbations in adult persistent asthma. J Allergy Clin Immunol Pract 2014; 2: 741-750.

17 Schatz M, Zeiger RS, Yang SJ, et al. Change in asthma control over time: predictors and outcomes. J Allergy Clin Immunol Pract 2014; 2: 59-64.

18 Jena AB, Ho O, Goldman DP, et al. The impact of the US Food and Drug Administration chlorofluorocarbon ban on out-of-pocket costs and use of albuterol inhalers among individuals with asthma. JAMA Intern Med 2015; 175: 1171-1179.

19 McRoy L, Weech-Maldonado R, Kilgore M. The relationship between direct to consumer advertising (DTCA) and asthma-related emergency department use among Medicaid-enrolled children. J Asthma 2014; 51: 922-926.

20 Wu AC, Butler MG, Li L, et al. Primary adherence to controller medications for asthma is poor. Ann Am Thorac Soc 2015; 12: 161-166. 
21 Tomasallo CD, Hanrahan LP, Tandias A, et al. Estimating Wisconsin asthma prevalence using clinical electronic health records and public health data. Am J Public Health 2014; 104: e65-e73.

22 Mukherjee M, Gupta R, Farr A, et al. Estimating the incidence, prevalence and true cost of asthma in the UK: secondary analysis of national stand-alone and linked databases in England, Northern Ireland, Scotland and Wales - a study protocol. BMJ Open 2014; 4: e006647.

23 Laforest L, Licaj I, Devouassoux G, et al. Asthma drug ratios and exacerbations: claims data from universal health coverage systems. Eur Respir J 2014; 43: 1378-1386.

24 Lemke LD, Lamerato LE, Xu X, et al. Geospatial relationships of air pollution and acute asthma events across the Detroit-Windsor international border: study design and preliminary results. J Expo Sci Environ Epidemiol 2014; 24: 346-357.

25 Jian ZH, Huang JY, Lin FCF, et al. The use of corticosteroids in patients with COPD or asthma does not decrease lung squamous cell carcinoma. BMC Pulm Med 2015; 15: 154.

26 Garne E, Hansen AV, Morris J, et al. Use of asthma medication during pregnancy and risk of specific congenital anomalies: a European case-malformed control study. J Allergy Clin Immunol 2015; 136: 1496-1502.

27 Tan NC, Nadkarni NV, Lye WK, et al. Ten-year longitudinal study of factors influencing nocturnal asthma symptoms among Asian patients in primary care. NPJ Prim Care Respir Med 2015; 25: 15064.

28 Kenyon CC, Rubin DM, Zorc JJ, et al. Childhood asthma hospital discharge medication fills and risk of subsequent readmission. J Pediatr 2015; 166: 1121-1127.

29 Rust G, Zhang S, Holloway K, et al. Timing of emergency department visits for childhood asthma after initial inhaled corticosteroid use. Popul Health Manag 2015; 18: 54-60.

30 von Bülow A, Kriegbaum M, Backer V, et al. The prevalence of severe asthma and low asthma control among Danish adults. J Allergy Clin Immunol Pract 2014; 2: 759-767.

31 Martin RJ, Price D, Roche N, et al. Cost-effectiveness of initiating extrafine- or standard size-particle inhaled corticosteroid for asthma in two health-care systems: a retrospective matched cohort study. NPJ Prim Care Respir Med 2014; 24: 14081 .

32 Schatz M, Meckley LM, Kim M, et al. Asthma exacerbation rates in adults are unchanged over a 5-year period despite high-intensity therapy. J Allergy Clin Immunol Pract 2014; 2: 570-574.

33 Capo-Ramos DE, Duran C, Simon AE, et al. Preventive asthma medication discontinuation among children enrolled in fee-for-service Medicaid. J Asthma 2014; 51: 618-626.

34 Nordlund B, Melén E, Schultz ES, et al. Prevalence of severe childhood asthma according to the WHO Respir Med 2014; 108: 1234-1237.

35 Ismaila A, Corriveau D, Vaillancourt J, et al. Impact of adherence to treatment with fluticasone propionate/ salmeterol in asthma patients. Curr Med Res Opin 2014; 30: 1417-1425.

36 Fung V, Graetz I, Galbraith A, et al. Financial barriers to care among low-income children with asthma: health care reform implications. JAMA Pediatr 2014; 168: 649-656.

37 Dilokthornsakul P, Chaiyakunapruk N, Schumock GT, et al. Calendar time-specific propensity score analysis for observational data: a case study estimating the effectiveness of inhaled long-acting beta-agonist on asthma exacerbations. Pharmacoepidemiol Drug Saf 2014; 23: 152-164.

38 Adimadhyam S, Schumock GT, Walton S, et al. Risk of arrhythmias associated with ipratropium bromide in children, adolescents, young adults with asthma: a nested case-control study. Pharmacotherapy 2014; 34: 315-323.

39 Blais L, Kettani FZ, Forget A. Associations of maternal asthma severity and control with pregnancy complications. J Asthma 2014; 51: 391-398.

40 Chang J, Freed GL, Prosser LA, et al. Comparisons of health care utilization outcomes in children with asthma enrolled in private insurance plans versus Medicaid. J Pediatr Health Care 2014; 28: 71-79.

41 Sullivan PW, Campbell JD, Ghushchyan VH, et al. Outcomes before and after treatment escalation to Global Initiative for Asthma steps 4 and 5 in severe asthma. Ann Allergy Asthma Immunol 2015; 114: 462-469.

42 Ali AK, Hartzema AG, Winterstein AG, et al. Application of multicategory exposure marginal structural models to investigate the association between long-acting beta-agonists and prescribing of oral corticosteroids for asthma exacerbations in the Clinical Practice Research Datalink. Value Health 2015; 18: 260-270.

$43 \mathrm{Wu}$ AC, Li L, Fung V, et al. Use of leukotriene receptor antagonists are associated with a similar risk of asthma exacerbations as inhaled corticosteroids. J Allergy Clin Immunol Pract 2014; 2: 607-613.

44 Tan CC, McDowell KM, Fenchel M, et al. Spirometry use in children hospitalized with asthma. Pediatr Pulmonol 2014; 49: 451-457.

45 Keast SL, Thompson D, Farmer K, et al. Impact of a prior authorization policy for montelukast on clinical outcomes for asthma and allergic rhinitis among children and adolescents in a state Medicaid program. J Manag Care Spec Pharm 2014; 20: 612-621.

46 Kim S, Kim J, Park SY, et al. Effect of pregnancy in asthma on health care use and perinatal outcomes. J Allergy Clin Immunol 2015; 136: 1215-1223.

47 Confino-Cohen R, Brufman I, Goldberg A, et al. Vitamin D, asthma prevalence and asthma exacerbations: a large adult population-based study. Allergy 2014; 69: 1673-1680.

48 Tunceli O, Williams SA, Kern DM, et al. Comparative effectiveness of budesonide-formoterol combination and fluticasone-salmeterol combination for asthma management: a United States retrospective database analysis. J Allergy Clin Immunol Pract 2014; 2: 719-726.

49 Bhattacharjee R, Choi BH, Gozal D, et al. Association of adenotonsillectomy with asthma outcomes in children: a longitudinal database analysis. PLoS Med 2014; 11: e1001753.

50 Nanchal R, Kumar G, Majumdar T, et al. Utilization of mechanical ventilation for asthma exacerbations: analysis of a national database. Respir Care 2014; 59: 644-653.

51 Tse SM, Charland SL, Stanek E, et al. Statin use in asthmatics on inhaled corticosteroids is associated with decreased risk of emergency department visits. Curr Med Res Opin 2014; 30: 685-693.

52 Sumino K, O'Brian K, Bartle B, et al. Coexisting chronic conditions associated with mortality and morbidity in adult patients with asthma. J Asthma 2014; 51: 306-314.

53 Li L, Vollmer WM, Butler MG, et al. A comparison of confounding adjustment methods for assessment of asthma controller medication effectiveness. Am J Epidemiol 2014; 179: 648-659. 
54 Hagiwara M, Delea TE, Stanford RH. Health-care utilization and costs with fluticasone propionate and fluticasone propionate/salmeterol in asthma patients at risk for exacerbations. Allergy Asthma Proc 2014; 35: 54-62.

55 Blais L, Kettani FZ, Forget A, et al. Asthma exacerbations during the first trimester of pregnancy and congenital malformations: revisiting the association in a large representative cohort. Thorax 2015; 70: 647-652.

56 Schneeweiss S, Avorn J. A review of uses of health care utilization databases for epidemiologic research on therapeutics. J Clin Epidemiol 2005; 58: 323-337.

57 Jorm L. Routinely collected data as a strategic resource for research: priorities for methods and workforce. Public Health Res Pract 2015; 25: e2541540.

58 Hemkens LG, Contopoulos-Ioannidis DG, Ioannidis JPA. Routinely collected data and comparative effectiveness evidence: promises and limitations. CMAJ 2016; 188: E158-E164.

59 Sheikh A, Cornford T, Barber N, et al. Implementation and adoption of nationwide electronic health records in secondary care in England: final qualitative results from prospective national evaluation in "early adopter" hospitals. BMJ 2011; 343: d6054-d6054.

60 Frenkel LD. Electronic health records - applications for the allergist/immunologist: all that glitters is not gold. Allergy Asthma Proc 2016; 37: 273-278.

61 Huzel L, Roos LL, Anthonisen NR, et al. Diagnosing asthma: the fit between survey and administrative database. Can Respir J 2002; 9: 407-412.

62 Manuel DG, Rosella LC, Stukel TA. Importance of accurately identifying disease in studies using electronic health records. BMJ 2010; 341: c4226.

63 Shivade C, Raghavan P, Fosler-Lussier E, et al. A review of approaches to identifying patient phenotype cohorts using electronic health records. J Am Med Inform Assoc 2014; 21: 221-230.

64 Springate DA, Kontopantelis E, Ashcroft DM, et al. ClinicalCodes: an online clinical codes repository to improve the validity and reproducibility of research using electronic medical records. PLoS One 2014; 9: e99825.

65 Wang SV, Verpillat P, Rassen JA, et al. Transparency and reproducibility of observational cohort studies using large healthcare databases. Clin Pharmacol Ther 2016; 99: 325-332.

66 Bel EH, Sousa A, Fleming L, et al. Diagnosis and definition of severe refractory asthma: an international consensus statement from the Innovative Medicine Initiative (IMI). Thorax 2011; 66: 910-917.

67 Chung KF, Wenzel SE, Brozek JL, et al. International ERS/ATS guidelines on definition, evaluation and treatment of severe asthma. Eur Respir J 2014; 43: 343-373.

68 Jacob C, Haas JS, Bechtel B, et al. Assessing asthma severity based on claims data: a systematic review. Eur J Health Econ 2017; 18: 227-241.

69 Ford ES. The epidemiology of obesity and asthma. J Allergy Clin Immunol Pract 2005; 115: 897-909.

70 Kotz D, Simpson CR, Sheikh A. Incidence, prevalence, and trends of general practitioner-recorded diagnosis of peanut allergy in England, 2001 to 2005. J Allergy Clin Immunol 2011; 127: 623-630.

71 Custovic A, Nicolaou N. Peanut allergy: overestimated in epidemiology or underdiagnosed in primary care? J Allergy Clin Immunol Pract 2011; 127: 631-632.

72 Panesar S, Javad S, de Silva D, et al. The epidemiology of anaphylaxis in Europe: a systematic review. Allergy 2013; 68: 1353-1361.

73 Nwaru BI, Mukherjee M, Gupta RP, et al. Challenges of harmonising data from UK national health surveys: a case study of attempts to estimate the UK prevalence of asthma. J R Soc Med 2015; 108: 433-439.

74 Mukherjee M, Wyatt JC, Simpson CR, et al. Usage of allergy codes in primary care electronic health records: a national evaluation in Scotland. Allergy 2016; 71: 1594-1602.

75 Howie L, Hirsch B, Locklear T, et al. Assessing the value of patient-generated data to comparative effectiveness research. Health Aff 2014; 33: 1220-1228. 